\title{
Clinical Knowledge and Practice of "Ketofol" at University of Gondar Comprehensive Specialized Hospital
}

\author{
Nigist Alemayehu Woldekidan ${ }^{1 *}$ and Ammas Siraj Mohammed ${ }^{2}$ \\ 1 Department of Clinical Pharmacy, School of Pharmacy, College of Medicine \& Health Sciences, University of Gondar, \\ Gondar, Ethiopia, ${ }^{2}$ Department of Clinical Pharmacy, School of Pharmacy, College of Health and Medical Sciences, \\ Haramaya University, Harar, Ethiopia
}

Background: Ketamine and propofol in a single syringe are reported to create an admixture used for balancing cardiorespiratory effects during induction of general anesthesia. This study aimed to assess the clinical practice and knowledge of "ketofol" among anesthesia providers.

Methods: A cross-sectional institutional-based study was conducted among anesthesia providers. Data abstracted format was prepared and distributed to senior anesthetists, junior anesthetist postgraduate students, and undergraduate students. The study was conducted from January 1, 2019, to January 30, 2019. Descriptive statistics and binary logistic regression were performed for frequency distribution and to determine

OPEN ACCESS

Edited by:

Somchai Amornyotin,

Mahidol University, Thailand

Reviewed by:

Mehmet Ali Erdogan,

Inönü University, Turkey Cemil Çolak,

Inönü University, Turkey

*Correspondence:

Nigist Alemayehu Woldekidan ngstalem14@gmail.com

Specialty section: This article was submitted to Intensive Care Medicine and Anesthesiology

a section of the journa

Frontiers in Medicine

Received: 27 April 2020 Accepted: 13 August 2021 Published: 28 September 2021

Citation: Woldekidan NA and Mohammed AS (2021) Clinical Knowledge and Practice of "Ketofol" at University of Gondar Comprehensive Specialized Hospital. Front. Med. 8:555973. doi: 10.3389/fmed.2021.555973 the association, respectively.

Result: From a total of 133 participants included in the study, the majority, 88 (66.2\%), were men and 75 (56.9\%) had 0-2 years of experience. More than two-thirds of participants, 105 (78.9\%), have never had a seminar or educational session about combined use. Lack of experience among 11 participants (8.3\%) was one of the reasons for not using "ketofol" followed by lack of knowledge among three (2.3\%) participants. The majority of participants, 112 (84.2\%), prefer ketamine and propofol to be administered one right after the other with separate syringes and the ratio to be 1:2, 64 (48.2). There was no significant association observed between sociodemographic and other characteristics and the practice of "ketofol."

Conclusion: In this study, nearly half of the participants rated their knowledge at the average level, and the study identifies that there is clinical knowledge and practice gap among anesthesia providers working in the University of Gondar Comprehensive Specialized Hospital (UOGCSH). Preparing educational sessions regarding "ketofol" for addressing identified barriers is among the recommendations forwarded to UOGCSH.

Keywords: anesthesia, ketamine, propofol, practice, knowledge

\section{INTRODUCTION}

Ketamine is a phencyclidine derivative, which is used as an intravenous anesthetic agent, and its mode of action is through causing dissociation anesthesia. Amnesic and analgesic effects, maintenance of muscle tone, protecting airway reflexes, and spontaneous respiration are among advantages that have been attributed to ketamine use. Nevertheless, different side effects were 
observed, including nausea, vomiting, raised blood pressure and heart rate, and development of hallucination, and also it was presumed to increase intracranial pressure $(1,2)$.

Propofol is neither opioid nor barbiturate, which is used for sedation and anesthesia providing rapid induction and recovery. Its mechanism of action involves the facilitation of inhibitory neurotransmission mediated by gamma-aminobutyric acid. Propofol has amnestic, anticonvulsant, and antiemetic properties. Its dose-related adverse effects include cardiovascular and respiratory depression (3-5).

Among the goals of procedural sedation and analgesia, the most common goals are achieving an adequate level of sedation with minimum pain and anxiety, increasing amnesia, decreasing drug-related adverse events, controlling behavior, and maintaining a stable cardiovascular and respiratory status procedural sedation and analgesia (PSA) (6). "Ketofol" has the advantage of reduced adverse effects of each drug counteracting the undesirable effects of one drug with the helpful effects of another. A combination of these drugs allows sedation to be achieved with a lower total dose of each drug, decreases the incidence of dose-related adverse effects, and ketamine can provide an analgesic effect that is absent when propofol is used alone. Because of this, physicians, nurses, and patients have shown a high level of satisfaction with its use $(7,8)$. This combination of safety and stability has also been proven (9).

A recent meta-analysis studied the safety and efficacy of ketofol in comparison with propofol as a single agent, which included six randomized control trials (RCTs), concluded that there was no statistically significant difference in safety. However, fewer respiratory events were documented in the combination group (10). Another meta-analysis by Jalili et al. (2016) corroborated the finding by revealing ketofol as a safe alternative to propofol for PSA (11). Moreover, the combination of ketamine and propofol appears to be safe in patients with cardiogenic shock, due to the lesser influence of ketamine on hemodynamic parameters (12). The recommended dosage is $0.5 \mathrm{mg} / \mathrm{kg}$ of a $1: 1$ mixture, followed by another dose of $0.5 \mathrm{mg} / \mathrm{kg}$. A dose of 0.25 $\mathrm{mg} / \mathrm{kg}$ is used afterward for maintenance of sedation (13).

As there is an increased use of this combination, the present study aims to assess the knowledge and practice of using "ketofol" admixture in clinical practice in the University of Gondar Comprehensive Specialized Hospital (UOGCSH). The second aim of this study is to assess whether age, sex, previous education, type of provider, and years of anesthetic experience affect their clinical practice and knowledge regarding "ketofol."

\section{METHOD}

\section{Study Area and Period}

The study was conducted from January 1, 2019, to January 30, 2019, at the UOGSCH on anesthesia providers. UOGSCH is one of the oldest teaching hospitals in Ethiopia, which is located in northwest Ethiopia. The hospital provides service for more than 7 million people who come from Gondar town and surrounding district areas.

Abbreviations: FDA, Food and Drug Administration; UOGCSH, University of Gondar Comprehensive Specialized Hospital; USA, United States of America.

\section{Study Design}

A hospital-based cross-sectional study was conducted on medical anesthesia fourth-year students and professionals who were practicing anesthesia at UOGCSH.

\section{Population}

The source population includes professionals who are responsible for administering anesthesia, whereas the study was conducted on anesthesia providers who were on practice sites during the study period.

\section{Inclusion and Exclusion Criteria}

All professionals who are responsible to administer anesthetic medication were included in the study whereas those who are not volunteered to participate in the study were excluded from the study.

\section{Sample Size Determination and Sampling Techniques}

All consented health professionals who were administering anesthesia, from fourth-year students (they are allowed to administer anesthesia under close supervision) to senior anesthetists, were considered due to a small number of the study population, and a convenience sampling method was used to recruit the study participants.

\section{Study Variables}

Clinical practice and knowledge about "ketofol" use were dependent variables whereas age, gender, work experience, and level of education were independent variables.

\section{Data Collection and Management}

The data were collected by two investigators through a selfadministered questionnaire. The data collection instrument was adapted from previous studies and consisted of sections focusing on Sociodemographic characteristics, the appropriate title of the participant, years of practicing anesthesia, and questions that assess the clinical knowledge and practice of ketamine-propofol (Ketofol) use. The data were collected from January 1, 2019, to January 30, 2019.

\section{Data Quality Assurance}

A standard questioner was adapted from previous study and prepared to make fit with our setup (14). The questionnaire was pre-tested on 10 anesthesia practitioners of other close-related setups. The pre-tested data were not included in the final analysis, and some amendments were done to avoid any ambiguity before the beginning of actual data collection. The data were collected using a structured self-administered questionnaire. Additionally, the confidentiality of the information obtained was secured in such a way that the information will not be disclosed to anyone in anyway and will only be used for the study purpose. Each variable had been coded before data entry.

\section{Data Compilation and Analysis}

After completing the data collection, the data were cleaned and checked for completeness. In addition, it was entered into the SPSS (IBM SPSS Statistics for Windows, Version 21.0; IBM Corp., Armonk, NY) for analysis. Sociodemographic and 
TABLE 1 | Background characteristics of anesthesia provider at the University of Gondar Comprehensive Specialized Hospital (UOGCSH), Gondar, Ethiopia, 2019.

\begin{tabular}{llc}
\hline Question & Response & $\boldsymbol{N}(\%)$ \\
\hline Age (year) & $19-22$ & $46(34.6)$ \\
& $23-26$ & $54(40.6)$ \\
& $27-30$ & $22(16.5)$ \\
& $31-34$ & $8(6.0)$ \\
& $>35$ & $3(2.3)$ \\
Gender & Male & $88(66.2)$ \\
Select your appropriate title & Female & $45(33.8)$ \\
& Senior anesthetist & $20(15)$ \\
& Postgraduate student & $25(18.8)$ \\
& Junior anesthetist & $6(4.5)$ \\
How many years have you & Undergraduate student & $82(61.7)$ \\
been practicing anesthesia? & 2-2years & $75(56.4)$ \\
& 5-10 years & $29(21.8)$ \\
& $>10 y e a r s$ & $25(18.8)$ \\
& $4(3)$ \\
\hline
\end{tabular}

other baseline information were summarized using descriptive statistics, and categorical variables were expressed as frequencies and percentages. For the sake of analysis, knowledge level was categorized into low and high (average and high), while practice was categorized into poor and good, depending on predefined criteria. Binary logistic regression was performed to assess the factors associated with clinical practice and knowledge about "ketofol" use.

\section{Ethical Considerations}

Ethical clearance was obtained from the ethics and research committee of the School of Pharmacy, the University of Gondar with the reference number of UoG-SoP/1164/2019. Informed oral consent was secured from the study participants prior to data collection. Information obtained from the study participants was kept confidential. Furthermore, participant identifiers were not used, and the data collected were used by the investigators only for the study.

\section{RESULT}

\section{Characteristics of Participants}

Among 150 participants, 140 responded to the questionnaire and among them seven were incomplete. The study population consist of $20(15 \%)$ senior anesthetists, $25(18.8 \%)$ postgraduate students, 6 (4.5\%) junior anesthetists, and 82 (61.7\%) undergraduate students.

Nearly half of the participants, 75 (56.4\%), had $0-2$ years of experience followed by 29 (21.8\%) from 2 to 5 years, 25 (18.8\%) from 5 to $10,4(3 \%) \geq 10$ years, and $88(66.2 \%)$, the majority of the participants, of them were men (Table 1).

\section{Knowledge About "Ketofol"}

A large number of participants, 116 (87.2\%), have heard and used ketamine and propofol combination 113 (85\%), and around half of the participants, 77 (57.9\%), rated their knowledge at an average level (Table 2).

More than two-thirds of the participants, 105 (78.9\%), have never had a seminar or educational session about the combination of ketamine-propofol, but in the last 1 year more than three-fourths of the participants, $113(85 \%)$, have used ketamine-propofol combination at the same time. Those who did not use in the last 1 year cited that the main reason is lack of experience, $11(8.3 \%)$, followed by lack of knowledge, 3 (2.3\%), and lack of propofol, $5(0.8 \%)$ (Table 2).

Regarding the question to list the main advantage of "ketofol", the answer of the respondent involves for hemodynamic stability of $64(48.1 \%)$, to decrease adverse effects to 51 (38.3\%) that occur when administered separately, to decrease the dose of each 15 (113\%). Almost half of the participants, 68 (51.13\%), do not know about the indication of "ketofol" and nearly more than one-third, $47(35.3 \%)$, put its indication as an induction agent (Table 2).

\section{The Preferred Ratio of Admixture}

The majority of participants, 112 (84.2\%), prefer ketamine and propofol to be administered one right after the other with separate syringes and the ratio to be 1:2, $64(48.2 \%)$.

\section{"Ketofol" Usage for Sedation and Induction}

More than one-third of participants, 90 (67.7\%), used the combination of ketamine and propofol for induction and rate the typical level of hemodynamic stability for induction on stable, 84 (63.2\%). Nearly half of the participants, 43 (32.3\%), did not use the combination as an induction agent and put lack of knowledge as a primary reason followed by lack of experience, 49 (36.8\%) (Table 2).

Around half of the participants, 62 (46.6\%), use "ketofol" for deep and moderate sedation/analgesia, 58 (43.6\%), and 60 (45.2\%) of participants have worries about the compatibility of the two drugs and are uncertain about the benefit of using the two drugs together, 22 (16.6\%).

Around three-fourth of participants, 72 (54.1\%), are very likely to attend if a seminar or educational session regarding current knowledge and practice of a combination of ketaminepropofol was offered (Table 2).

Binary logistic regression was performed to identify the association of sociodemographic and other characteristics with the practice of "ketofol." We have assessed the association between the sociodemographic and other characteristics, and the practices including the preferred ratio of administration (1:1 vs. others), a clinical practice that is usually a combination of ketamine and propofol used (induction vs. sedation), and preferred administration combination (mixing in the same syringe vs. other administration). However, each of the dependent variables has no significant association with the sociodemographic and other characteristics. 
TABLE 2 | Knowledge and practice of ketamine-propofol combination (ketofol) among anesthesia providers at the UOGCSH, Gondar, Ethiopia, 2019.

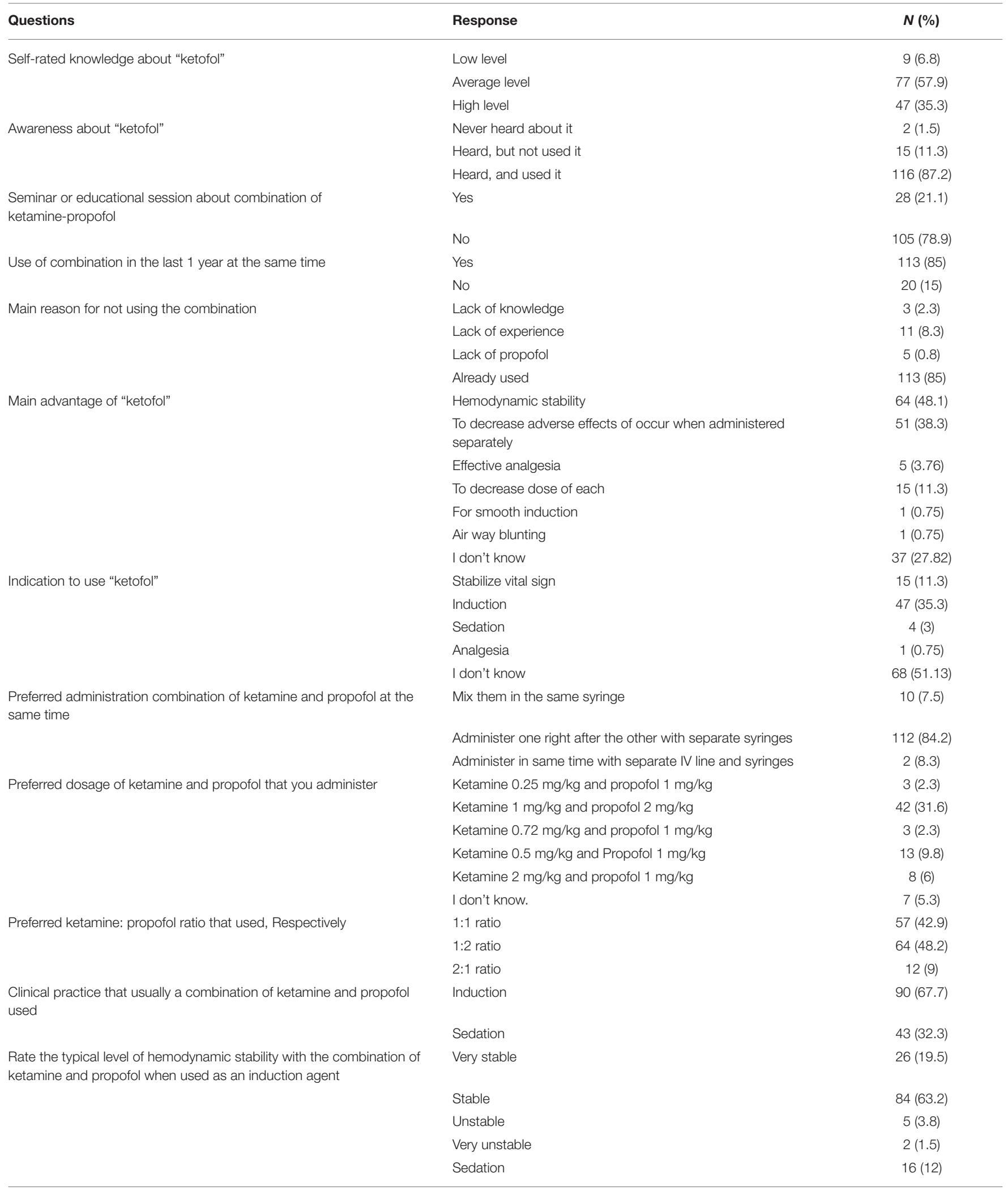


TABLE 2 | Continued

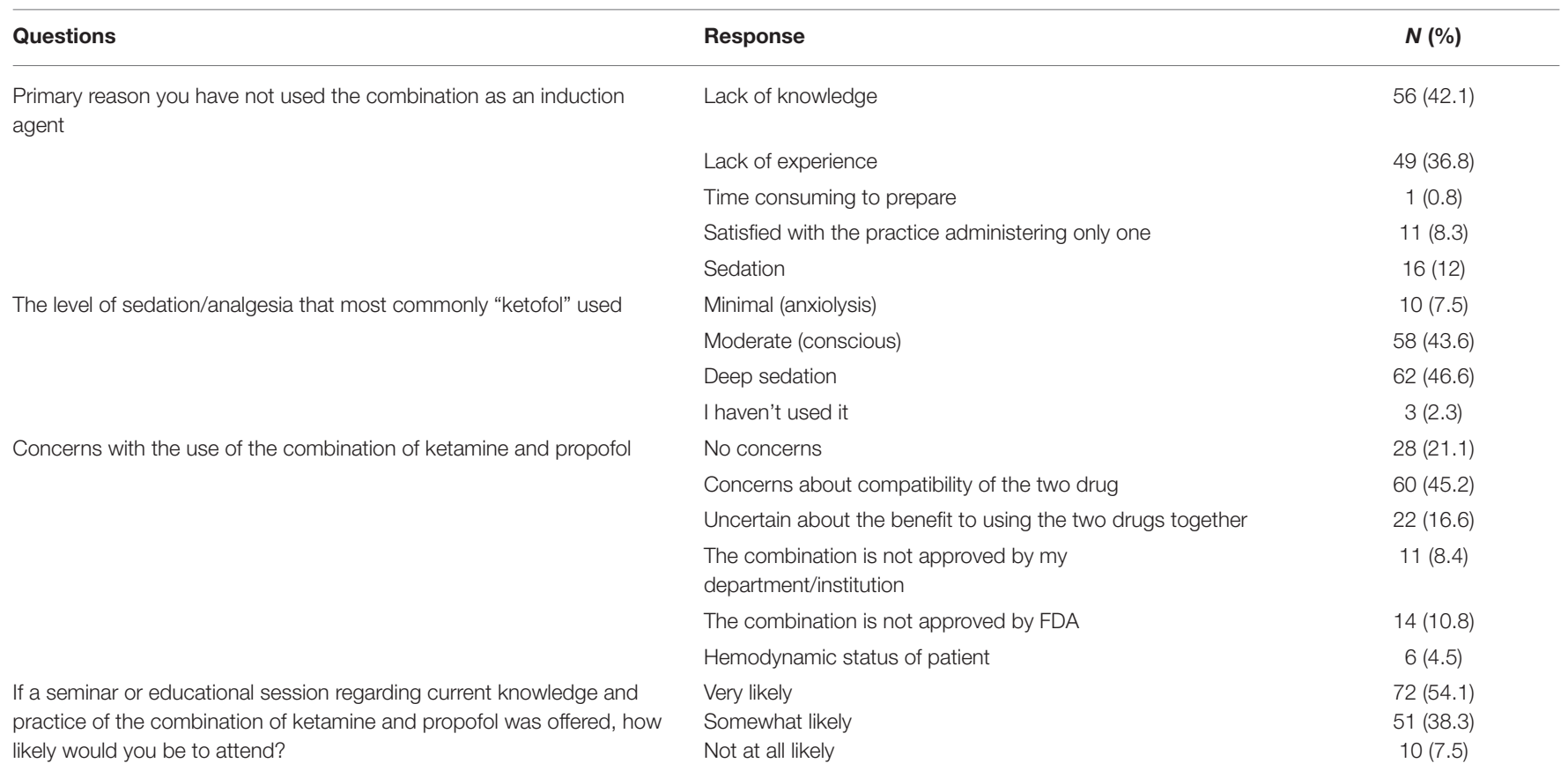

\section{DISCUSSION}

A combination of ketamine and propofol (Ketofol) has been proven to be effective in the operating room, ambulatory setting, and emergency department mixed in the same syringe (15-18).

A remarkable number of $116(87.2 \%)$ of the respondents has used this combination drug and rated their level of knowledge about "ketofol" at an average of 77 (57.9\%). Few numbers, $3(2.3 \%)$, of participants, have raised lack of knowledge as a reason for not using combination drugs, but it was the main reason for most of the participants in the study conducted in the USA (14).

Most of the respondents, 112 (84.2\%), prefer administration of ketamine and propofol to be administered one right after the other with separate syringes and with the ratio of $1: 2,64(48.2 \%)$. Preference of mixing was significantly associated with the year of experience ( $p=0.003)$ and the level of study of a professional $(p=0.004)$. In the previous study, single-syringe "ketofol" in a 1:1 ratio for sedation and analgesia of adults in the emergency department resulted in quick recovery, few adverse events, and patient and staff satisfaction (18).

In our study, there was no significant association observed between the year of experience and clinical use of "ketofol." However, in another study, the use of "ketofol" for both sedation and anesthesia was associated with years of experience $(7,19)$.

Most of the respondents, 84 (63.2\%), rate the level of hemodynamic stability as stable when the combination of ketamine and propofol is used as an induction agent. Even if the emergence of delirium is less prevalent, hemodynamic stability may be further required than the worrisome consequence of the condition $(7,18-20)$.

Almost half of the participants, 72 (54.1\%), are very likely to attend a seminar or educational sessions regarding current knowledge and practice of the combination of ketamine and propofol. Similarly, the study in Minnesota, USA, shows the education had a statistical impact on the concern regarding the admixture. Also, web-based and face-to-face education have proven to be effective if required or mandatory. These factors of education have been studied in both nursing and other medical professions, with similar conclusions (14).

\section{CONCLUSION}

This is the first attempt to assess clinical knowledge and practice of "ketofol" in UOGCSH. A large number of participants prefer ketamine and propofol to be administered one right after the other with separate syringes and the ratio to be $1: 2$, and nearly half of the participants rated their level of knowledge as average. Preference for mixing was significantly associated with the year of experience and the level of study of a professional. This study identifies clinical practice and knowledge differences among anesthesia providers of UOGCSH. As it is a critical area of patient care, the institution should prepare educational sessions to promote evidencebased medicine so that practitioners will update themselves by reviewing articles regarding "ketofol" admixture for the best care of the patient. 


\section{DATA AVAILABILITY STATEMENT}

The original contributions presented in the study are included in the article/supplementary material, further inquiries can be directed to the corresponding author/s.

\section{ETHICS STATEMENT}

The studies involving human participants were reviewed and approved by Ethics and research committee of School of Pharmacy and the Clinical Directorate of University

\section{REFERENCES}

1. Green SM, Krauss B. The semantics of ketamine. Ann Emerg Med. (2000) 36:480-2. doi: 10.1067/mem.2000.109510

2. Strayer RJ, Nelson LS. Adverse events associated with ketamine for procedural sedation in adults. Am J Emerg Med. (2008) 26:985-1028. doi: 10.1016/j.ajem.2007.12.005

3. Claeys MA, Gepts E, Camu F. Haemodynamic changes during anaesthesia induced and maintained with propofol. Br J Anaesth. (1988) 60:39. doi: $10.1093 / \mathrm{bja} / 60.1 .3$

4. Bahn EL, Holt KR. Procedural sedation and analgesia: a review and new concepts. Emerg Med Clin North Am. (2005) 23:503-17. doi: 10.1016/j.emc.2004.12.013

5. Mirrakhimov AE, Voore P, Halytskyy O, Khan M, Ali AM. Propofol Infusion Syndrome in Adults: A Clinical Update. Crit Care Res Pract. (2015) 2015:10. doi: 10.1155/2015/260385

6. Kliegman R, Stanton B, Behrman RE, St. Geme JW, Schor NF, Nelson WE. Nelson textbook of pediatrics. (2016) 2.

7. Willman EV, Andolfatto G, A. prospective evaluation of "ketofol" (ketamine/propofol combination) for procedural sedation and analgesia in the emergency department. Ann Emerg Med. (2007) 49:23-30. doi: 10.1016/j.annemergmed.2006.08.002

8. Alletag MJ, Auerbach MA, Baum CR. Ketamine, propofol, and ketofol use for pediatric sedation. Pediatric Emergency Care. (2012) 28:13915. doi: 10.1097/PEC.0b013e318276fde2

9. Friedberg B. Propofol-ketamine technique: dissociative anesthesia for office surgery (A 5-year review of 1264 cases) Aesthetic Plast Surg. (1999) 23:705. doi: 10.1007/s002669900245

10. Yan JW, McLeod SL, Iansavitchene A. Ketamine-propofol versus propofol alone for procedural sedation in the emergency department: a systematic review and meta-analysis. Acad Emerg Med. (2015) 22:1003-13. doi: 10.1111/acem.12737

11. Jalili M, Bahreini M, Doosti-Irani A, et al. Ketamine-propofol combination (ketofol) vs propofol for procedural sedation and analgesia: systematic review and meta-analysis. Am J Emerg Med. (2016) 34:558-69. doi: 10.1016/j.ajem.2015.12.074

12. Li L, Vlisides PE. Ketamine: 50 years of modulating the mind. Front Hum Neurosci. (2016). 10:612. doi: 10.3389/fnhum.2016.00612

13. Lamperti M. Adult procedural sedation: an update. Curr Opin Anaesthesiol. (2015) 28:662-7. doi: 10.1097/ACO.0000000000000244

14. Olson AN, Rao WR, Marienau ME, Smischney NJ. Period prevalence of ketamine-propofol admixture "ketofol" in the operating room among anesthesia providers at an academic medical center. Med Sci Monit Int Med J Exp Clin Res. (2015) 21:1737-44. doi: 10.12659/MSM.893944 of Gondar Comprehensive Specialized Hospital.. Written informed consent for participation was not required for this study in accordance with the national legislation and the institutional requirements.

\section{AUTHOR CONTRIBUTIONS}

All authors listed have made a substantial, direct and intellectual contribution to the work, and approved it for publication.

15. Camu F, Vanlersberghe C. Pharmacology of systemic analgesics. Best Pract Res Clin Anaesthesiol. (2002) 16:475-88. doi: 10.1053/bean.2002.0262

16. Frey K, Sukhani R, Pawlowski J, Pappas AL, Mikat-Stevens M, Slogoff S. Propofol versus propofol-ketamine sedation for retrobulbar nerve block: comparison of sedation quality, intraocular pressure changes, and recovery profiles. Anesth Analg. (1999) 89:317-21. doi: 10.1213/00000539-199908000-00013

17. Mortero RF, Clark LD, Tolan MM, Metz RJ, Tsueda K, Sheppard RA. The effects of small-dose ketamine on propofol sedation: respiration, postoperative mood, perception, cognition, and pain. Anesth Analg. (2001) 92:14659. doi: 10.1097/00000539-200106000-00022

18. Gallo de Moraes A, Racedo Africano CJ, Hoskote SS, Reddy DRS, Tedja R, Thakur L, et al. Ketamine and propofol combination ("ketofol") for endotracheal intubations in critically ill patients: a case series. Am J Case Reports. (2015) 16:81-6. doi: 10.12659/AJCR.8 92424

19. Smischney NJ, Beach ML, Loftus RW, Dodds TM, Koff MD. Ketamine/propofol admixture (ketofol) is associated with improved hemodynamics as an induction agent: a randomized, controlled trial. J Trauma Acute Care Surg. (2012) 73:94101. doi: 10.1097/TA.0b013e318250cdb8

20. Andolfatto G, Willman E, A. prospective case series of single-syringe ketamine-propofol (Ketofol) for emergency department procedural sedation and analgesia in adults. Academic Emergency Med. (2011) 18:23745. doi: 10.1111/j.1553-2712.2011.01010.x

Conflict of Interest: The authors declare that the research was conducted in the absence of any commercial or financial relationships that could be construed as a potential conflict of interest.

Publisher's Note: All claims expressed in this article are solely those of the authors and do not necessarily represent those of their affiliated organizations, or those of the publisher, the editors and the reviewers. Any product that may be evaluated in this article, or claim that may be made by its manufacturer, is not guaranteed or endorsed by the publisher.

Copyright (c) 2021 Woldekidan and Mohammed. This is an open-access article distributed under the terms of the Creative Commons Attribution License (CC BY). The use, distribution or reproduction in other forums is permitted, provided the original author(s) and the copyright owner(s) are credited and that the original publication in this journal is cited, in accordance with accepted academic practice. No use, distribution or reproduction is permitted which does not comply with these terms. 